\title{
Mechanical analysis of bi-component-fibre nonwovens: finite-element strategy
}

Farukh Farukh ${ }^{(1)}$, Emrah Demirci ${ }^{(1)}$, Baris Sabuncouglu ${ }^{(1)}$, Memiş Acar ${ }^{(1)}$, Behnam Pourdeyhimi (2) $*$ Vadim V. Silberschmidt ${ }^{(1)}$

1 - Wolfson School of Mechanical and Manufacturing Engineering, Loughborough University, UK 2 - Nonwovens Cooperative Research Center, North Carolina State University, Raleigh, NC, USA *Corresponding author's e-mail: V.Silberschmidt@lboro.ac.uk

Tell: +441509227504 ; fax: +441509227502

Add: Wolfson School of Mechanical and Manufacturing Engineering, Loughborough University, UK

Keywords: non-woven fabrics; composite; microstructure; bicomponent; finite element

\begin{abstract}
:
In thermally bonded bi-component fibre nonwovens, a significant contribution is made by bond points in defining their mechanical behaviour formed as a result of their manufacture. Bond points are composite regions with a sheath material reinforced by a network of fibres' cores. These composite regions are connected by bi-component fibres — a discontinuous domain of the material. Microstructural and mechanical characterisation of this material was carried out with experimental and numerical modelling techniques. Two numerical modelling strategies were implemented: (i) traditional finite element (FE) and (ii) a new parametric discrete phase FE model to elucidate the mechanical behaviour and underlying mechanisms involved in deformation of these materials. In FE models the studied nonwoven material was treated as an assembly of two regions having distinct microstructure and mechanical properties: fibre matrix and bond points. The former is composed of randomly oriented core/sheath fibres acting as load-transfer link between composite bond points. Randomness of material's microstructure was introduced in terms of orientation distribution function (ODF). The ODF was obtained by analysing the data acquired with scanning electron microscopy (SEM) and X-ray micro computed tomography $(\mathrm{CT})$. Bond points were treated as a deformable two-phase composite. An in-house algorithm was used to calculate anisotropic material properties of composite bond points based on properties of constituent fibres and manufacturing parameters such as the planar density, core/sheath ratio and fibre diameter. Individual fibres
\end{abstract}


connecting the composite bond points were modelled in discrete phase model directly according to their orientation distribution. The developed models were validated by comparing numerical results with experimental tensile test data, demonstrating that the proposed approach is highly suitable for prediction of complex deformation mechanisms, mechanical performance and structure-properties relationships of composites.

\section{Introduction}

Thanks to their beneficial material properties, such as, high formability and improved resistance to impact, interest in application of textile composites is continuously increasing, for instance, in automotive and aerospace industry [1]. As compared to woven composites, the nonwoven composites have been getting more attention, which is evidenced by a rapidly growing number of patents in recent decades. The reason for this attention from academia and industry lies in several advantages attributed to nonwoven composites such as low cost and quicker manufacturing of nonwovens along with better control on fibres orientation-based properties [2]. Despite this importance and enormous research in this field, there is no adequate design tool for nonwoven composites. One of the main challenges in this is insufficient understanding of a complex mechanical behaviour of the nonwoven fabric [3-5] especially in thermally bonded bi-component fibre nonwovens, in which bond points behave like a composite themselves. Therefore, this research is focussed on mechanical behaviour of thermally bonded bicomponent fibres nonwovens.

Such materials can be manufactured by embedding a nonwoven web, bonded with any of three main techniques: chemical, mechanical or thermal, into a matrix to achieve a necessary combination of properties. This study is focussed on a type of nonwovens, which were thermally bonded by calendering using core-sheath type bi-component fibres as shown in Fig. 1. Thermal bonding is the most widely used technique for manufacturing of nonwovens $[2,6]$. During thermal bonding, fibres are passed through hot calendar rolls with smooth or embossed surface design. Bonding occurs at raised areas of the embossed calender under pressure and high temperature by partial melting and 
subsequent solidification of fibres. The molten sheath material that has a lower melting point acts as adhesive while core parts of the fibres remain fully intact in the bond spots, thus forming them with a two-phase composite structure with matrix and fibre reinforcement. The bonding process results in two distinct regions, namely, bond points and a fibre matrix, as shown in Fig. 2. The same technique with smooth calenders can be used for the manufacturing of composite sheets using nonwovens as precursor [7]. Therefore, this study is focussed on calendar-bonded nonwovens.

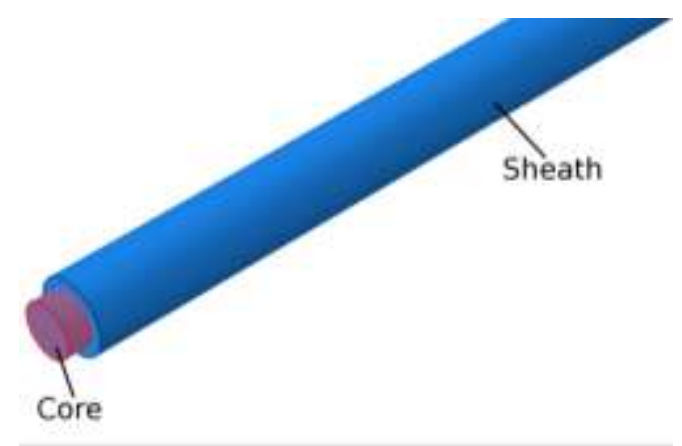

Fig. 1. Structure of core/sheath type bi-component fibre

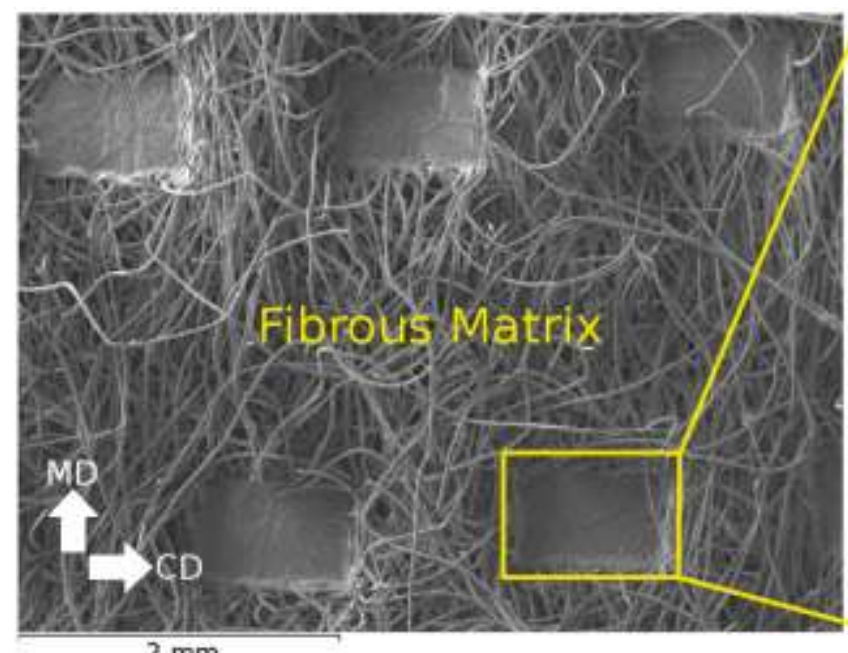

$2 \mathrm{~mm}$

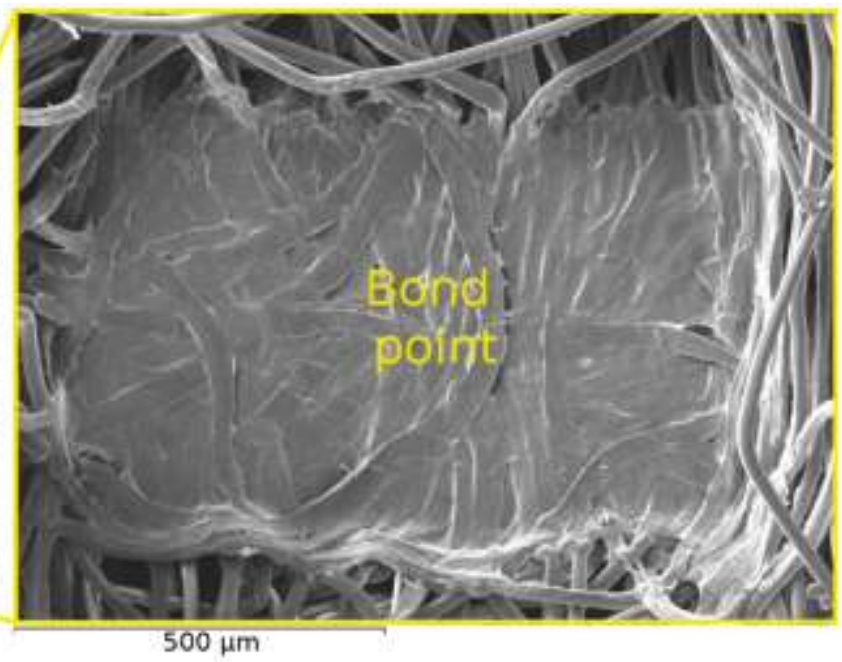

(b)

Fig. 2. SEM images of bi-component fibre nonwoven fabric composed of composite bond points (b) and fibrous matrix (a)

Due to a multi-scale nature of nonwovens, both continuous and discrete modelling approaches to their analyses were used in the literature. Continuous phase models provide the benefit of using a standard finite element method. Still, they provide a macroscopic response of the material without 
sufficient information about underlying mechanisms defining their mechanical behaviour [8-10]. In contrast to continuous phase models, discrete models of nonwovens were applied at the level of unit cell in combination with homogenization approach $[11,12]$. These models do not represent the microstructure reslistically and cannot be used to understand the structure-properties relationship of nonwovens. Other discrete phase models developed by the authors employed direct introduction of individual fibres according to the experimentally measured orientation distribution [13-17]. These models incorporate randomness in structure of nonwovens into the model to predict their anisotropic behaviour. As a result, they are capable to provide information about structural evolution and other main aspects of the fabric's mechanical behaviour. All these models were either developed to deal with mono-component fibre nonwovens or based on a traditional continuous phase modelling technique, which could predict only a macroscopic response of the composite fabrics. To the authors' knowledge, none of the model in literature based on discreet modelling deal with thermally bonded bi-component fibre nonwovens, which make a special type of structure consisting of composite domains connected by a network of fibres.

In this paper, a novel modelling strategy based on a discrete phase modelling approach is presented to characterise the mechanical behaviour of textile composite nonwovens formed by bi-component fibres. The modelling process started with determining morphological and mechanical properties of the fabric and its constituent fibres. Bond points were modelled as continuous structure, with their mechanical properties obtained by a special developed in-house algorithm. Fibres between the bond points were modelled directly according to their orientation distribution forming the discontinuous part of the model. This approach is juxtaposed with simulations employing a traditional FE model.

\section{Experimentation}

\subsection{Fabric Behaviour}

The morphological features and mechanical behaviour of a $35 \mathrm{~g} / \mathrm{m}^{2}$ bi-component fibre nonwoven, which is a focus of our study, were obtained by performing various analysis and tests. The fabric 
was scanned with X-ray Micro Computed Tomography (CT) and observed with SEM to acquire the information about its microstructure. The data about the size, shape and pattern of bond points and thickness of fibrous matrix and bond points were collected directly from the captured images. The bond points of the fabric were similar to those in Fig. 2. Table 1 provide information about the measured size, shape and pattern of bond points. In the images of the fabric at micro level, it was observed that the diameter of the fibres was not constant along their length; this variation is a result of the manufacturing process.

Table 1. Parameters of geometry (in $\mathrm{mm}$ ) and pattern of bond points

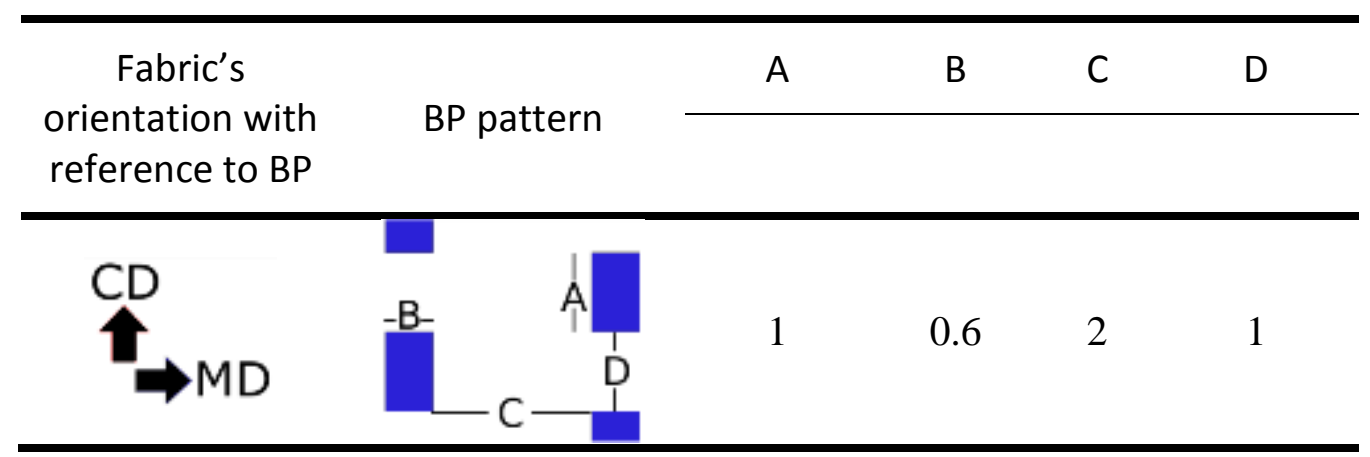

Mechanical characterization of the fabric was implemented employing uni-axial tensile tests on rectangular coupons of the fabric with $15 \mathrm{~mm}$ x $10 \mathrm{~mm}$ dimensions using cut-strip grab test method [18]. In order to capture anisotropy of the material, tensile tests were performed in MD and CD directions. The results of uni-axial tests in terms of force-displacement curves are given in Fig. 3 demonstrating a scatter in experimental results due to random microstructure of the fabric. The deformed shape of the fabric at extension of 50\% is shown in Fig. 4. At that level of fabric's extension, misalignment of bond points e.g. breaks of bond points lines in the initial pattern can be observed. The reason for this is a random orientation distribution of fibres. One of the main deformation process of nonwoven is re-orientation of fibres towards the loading direction during extension [16, 17], and their final position depend upon their initial orientation, leading to randomness in displacement of bond points. 


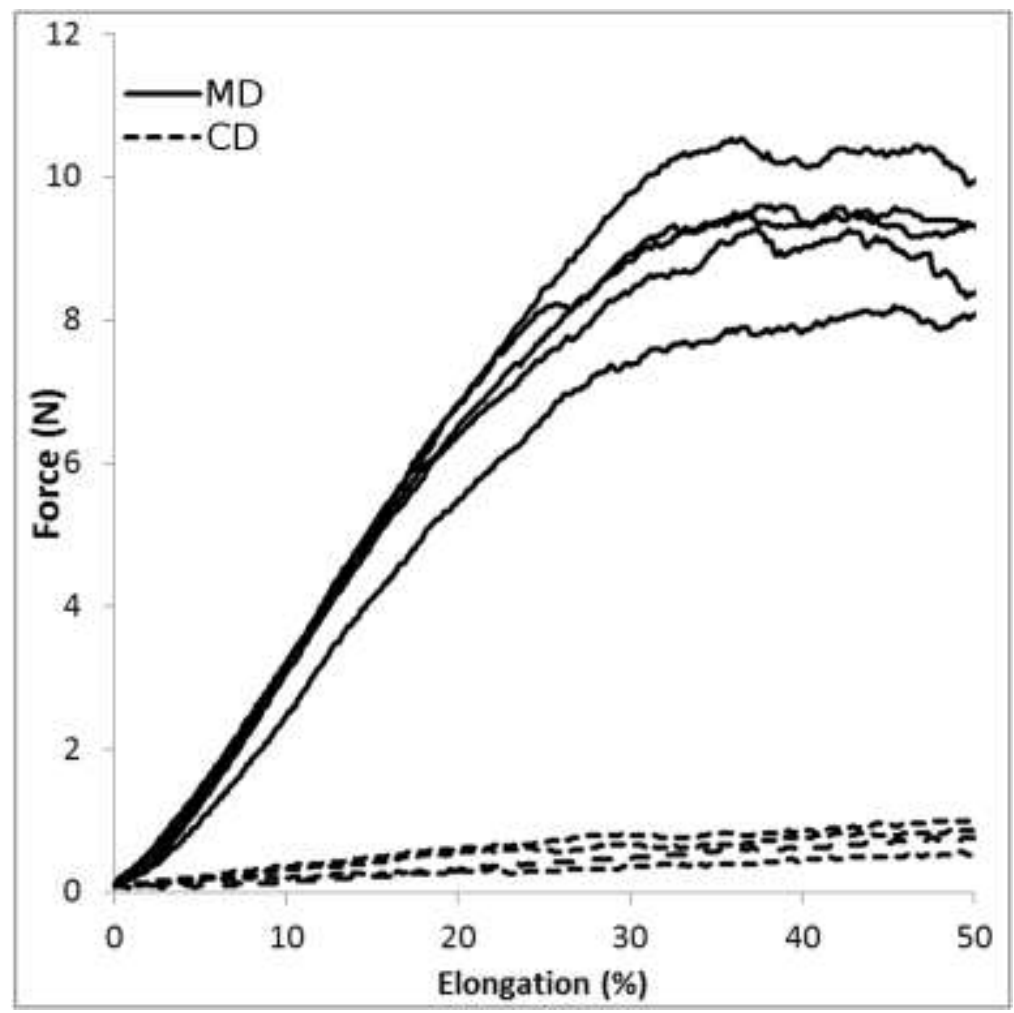

Fig. 3. Mechanical behaviour for PP/PE $35 \mathrm{~g} / \mathrm{m}^{2}$ thermally bonded nonwoven in MD and CD (dimensions of specimens: $15 \mathrm{~mm} \times 10 \mathrm{~mm}$ )

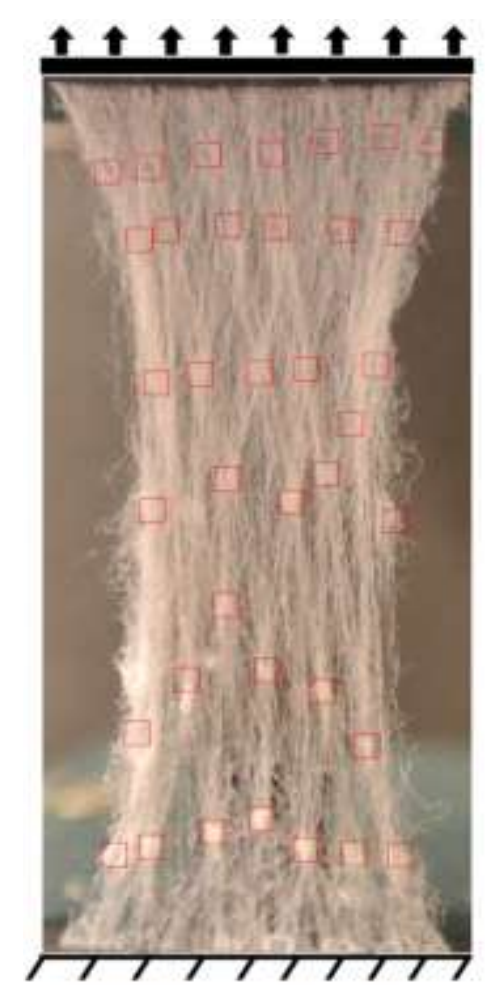

Fig. 4. Deformed shape of fabric at 50\% extension in MD showing misalignment of bond points (red squares) 


\subsection{Single-fibre Behaviour}

Fibres are a basic constituent of the fabric and play an important role in determination of its mechanical properties. The type of bi-component fibre nonwoven in this study is composed of core/sheath type fibres (Fig. 1) with the polymer used for their core region having a higher value of the elasticity modulus than the one of sheath region.

Generally, the mechanical properties (elastic-plastic behaviour) of a single fibre can be obtained by performing tensile tests on fibres and its mechanical behaviour can be derived from that of its core and sheath regions using the Rule of Mixture (RoM). In thermally calendered bonded nonwovens, fibres are passed through hot calendar rolls with smooth or embossed surface design. Bonding occurs at raised areas of the embossed calender under pressure and high temperature by partial melting and subsequent solidification of fibres. Since high temperature variation is involved in thermal bonding process which affects the mechanical behaviour of polymeric fibres, therefore, using the Rule of Mixture (RoM) with the polymer properties available in literature to compute the mechanical behaviour of fibres is not suitable. The mechanical properties of the fibres within thermally bonded nonwovens are obtained experimentally.

As a typical example, a Polypropylene/Polyethylene (PP/PE) core/sheath bi-compoent fibre with diameter of $23 \mu \mathrm{m}$, extracted from a free edge of $35 \mathrm{~g} / \mathrm{m}^{2}$ of fabric is studied here. Each of both materials - PP and PE — occupy 50\% of the cross-sectional area of the fibre. Instron Micro Tester 5848 was used to perform the single-fibre tensile tests; the details of procedure are given in [15]. Figure 5 shows a typical behaviour of polymer bi-component fibres obtained with the tensile tests at different strain rates. The difference in curves is linked to viscous properties in the material. Since fibres of the network between the bond points were modelled explicitly, the properties obtained with the single-fibre tensile tests were directly assigned to them. The influence of singlefibre material properties on the mechanical behaviour of the nonwoven network was incorporated by assuming fibres as isotropic, elastic-plastic with piece-wise linear hardening. The total strain of the fibre was a combination of elastic and plastic contributions. In the elastic region, stresses in the 
fibres as a function of strain can be obtained by relating these two using the elastic modulus, whereas the von Mises yield criterion was used for the onset of plastic deformation. the incremental stress-strain relation in fibres in elastic region is given as (Simo and Hughes, 1997; MSC. Marc, 2013):

$$
\sigma_{e}^{n+1}=\sigma_{e}^{n}+E \Delta \varepsilon_{e}^{n+1}
$$

where $E$ is the Young's modulus. Using the flow stress definition based on table-based input, the hardening slope at each increment is obtained by numerically differentiating the values given in the table; these values are based on a plot of the stress versus plastic strain for a tensile test. The generalized form of the work-hardening coefficient has the following form:

$$
H=\frac{d \bar{\sigma}}{d \bar{\varepsilon}^{\mathrm{p}}}
$$

where $d \bar{\varepsilon}^{\mathrm{p}}$ and $d \bar{\sigma}$ are equivalent plastic strain and equivalent stress. The time-dependent behaviour of the fibre was modelled by using single-fibre creep data in conjunction with plasticity. The equivalent creep strain-rate was then assumed to be a piece-wise linear approximation, expressed as:

$$
\dot{\varepsilon}_{\text {creep }}=A \bar{\sigma} \cdot \bar{\varepsilon} \cdot \frac{d k(t)}{d t}
$$

where $A$ is a constant; $\bar{\sigma}$ is equivalent stress, $\bar{\varepsilon}^{c}$ equivalent creep strain and $t$ is time. The details of material modelling process are given elsewhere $[17,19,23]$. The properties of composite bond points consisting of core fibres and sheath matrix were computed with a specially developed Matlab code, which is discussed in next section. 


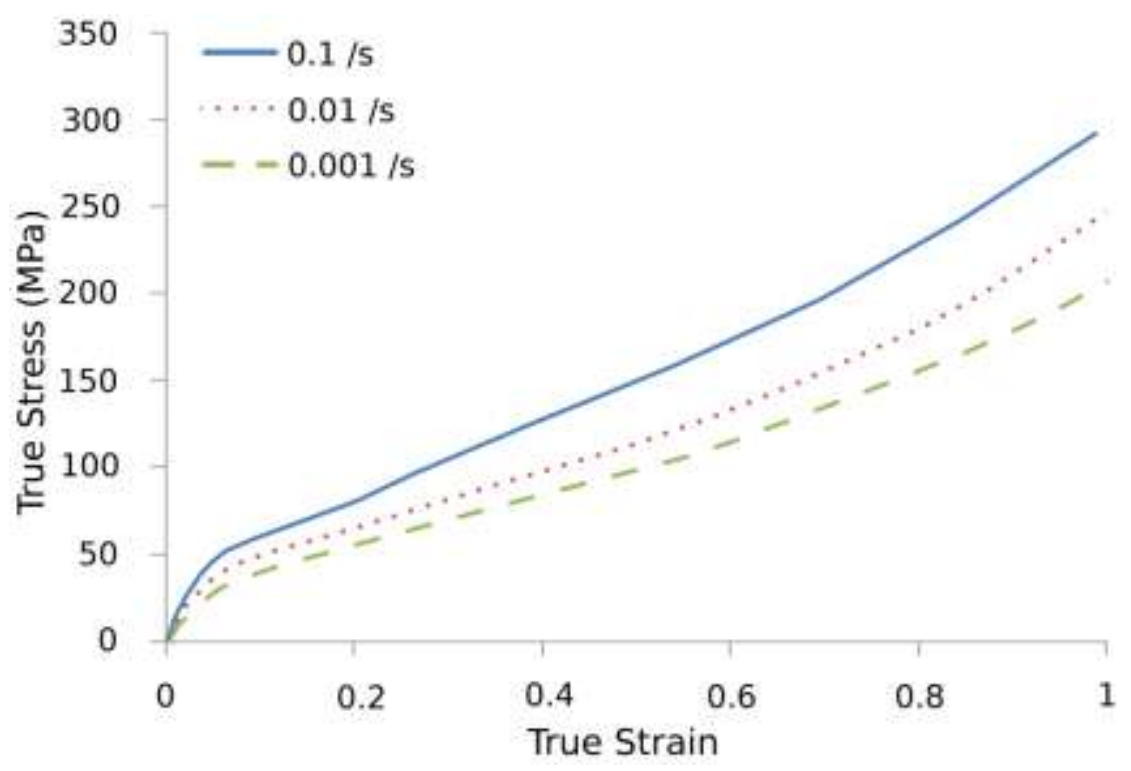

Fig. 5. Mechanical behaviour of PP/PE 50/50 fibre of $23 \mu \mathrm{m}$ diameter for three strain rates

\section{Input to finite-element model}

\subsection{Anisotropy}

Anisotropy of nonwovens is an unavoidable phenomenon, thanks to a non-uniform orientation distribution of their fibres. This anisotropy must be taken into account in simulations of nonwovens to predict their direction-dependent mechanical response. An orientation distribution function (ODF) for fibres in the studied nonwoven was obtained employing an algorithm based on the Hough transform written in Matlab. The ODF of PP/PE $35 \mathrm{~g} / \mathrm{m}^{2}$ was computed based on the SEM image of a fibre-matrix region and given in Fig. 6. The details of computation for the ODF are given in [20]. As expected, the calculated distribution of fibres (Fig. 6) demonstrates their preferential orientation along MD. 


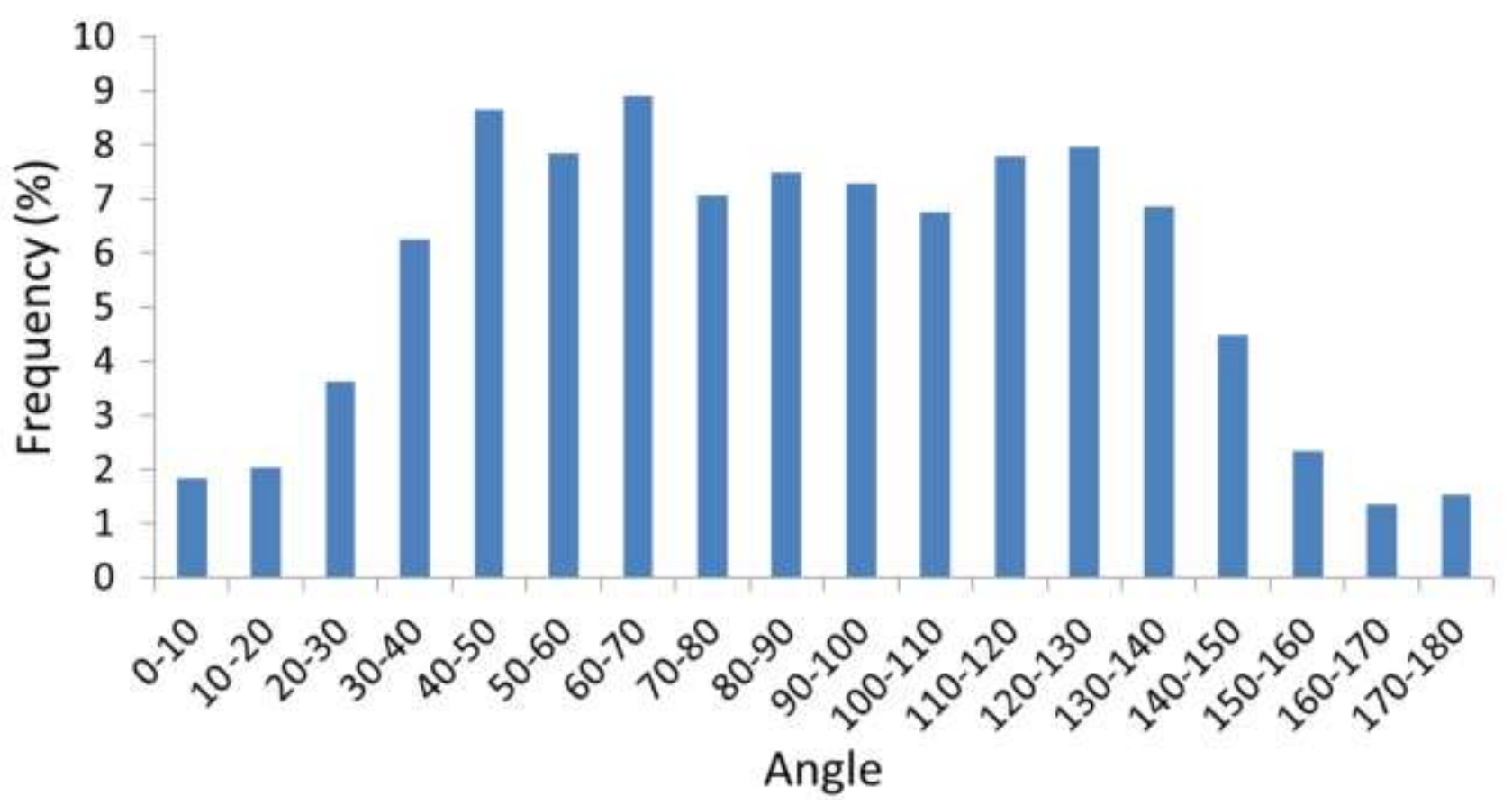

Fig. 6. ODF of PP/PE $35 \mathrm{~g} / \mathrm{m}^{2}$ thermally bonded nonwoven $\left(90^{\circ}\right.$ corresponds to $\mathrm{MD} ; 0^{\circ}$ and $180^{\circ}$ corresponds to $\mathrm{CD}$ )

\subsection{Mechanical Properties}

Thermally calendered bonded nonwoven consists of two regions — bond points and fibrous matrix — as shown in Fig. 2; the properties of both regions are required for successful implementation of the finite-element model. The mechanical properties of the fibrous matrix were obtained based on the single-fibre tensile tests. Since fibres were introduced explicitly into the model and carry only the tensile load, as discussed in modelling section, the properties obtained from the single-fibre tensile tests such as the Young's modulus and flow curve were used to model the behaviour of fibres in the matrix region. However, the properties of composite bond points were obtained by using a specially developed Matlab code. In fabric, a bond point is attached to various fibres and can undergo loading in various directions at a time, therefore, only the tensile data obtained from uniaxial single-fibre tests is not sufficient to model the directional dependent material behaviour of composite bond points. Therefore, RoM is used to obtain the properties of bond points.

Additionally, the properties of composite bond points are characterised by the orientation distribution of fibres which is also considered while computing the properties of bond points. The input parameters required to assess the mechanical properties of composite bond points based on 
several types of data such as manufacturing parameters, properties of constituent fibres and properties related to raw form of polymers used for core and sheath material. The full list of the required paramerts is given in Table 2.

Table 2. Input parameters for Matlab code to compute bond point properties

\begin{tabular}{|c|c|c|}
\hline Manufacturing parameters & $\begin{array}{l}\text { Properties of core and } \\
\text { sheath materials }\end{array}$ & $\begin{array}{c}\text { Properties of single } \\
\text { fibre }\end{array}$ \\
\hline Nonwoven Planar density & Density & \\
\hline Sheath cross-sectional ratio & Young's modulus & Young's modulus \\
\hline CMD and CCD & Poisson’s ratio & Flow curve \\
\hline Fibre diameter & Shear modulus & \\
\hline Calendaring temperature & Melting temperature & \\
\hline
\end{tabular}

In order to calculate these properties, first, the level of orthotropy based on the ODF was calculated by using the following equations:

$$
\begin{aligned}
& C_{\mathrm{MD}}=\frac{\sum_{i=1}^{N}\left|\sin \alpha_{i}\right|}{\sum_{i=1}^{N}\left|\sin \alpha_{i}\right|+\sum_{i=1}^{N}\left|\cos \alpha_{i}\right|}, \\
& C_{\mathrm{CD}}=\frac{\sum_{i=1}^{N}\left|\cos \alpha_{i}\right|}{\sum_{i=1}^{N}\left|\sin \alpha_{i}\right|+\sum_{i=1}^{N}\left|\cos \alpha_{i}\right|},
\end{aligned}
$$

where $\mathrm{C}_{\mathrm{MD}}$ and $\mathrm{C}_{\mathrm{CD}}$ are the parameters defining the level of orthotropy with regard to $\mathrm{MD}$ and $\mathrm{CD}$ (obviously, $\mathrm{MD}+\mathrm{CD}=1$ ), $\alpha_{i}$ is the angle between the $i$ th fibre and $\mathrm{CD} ; N$ is the total number of fibres used for the calculation of ODF. With these parameters $\left(\mathrm{C}_{\mathrm{MD}}\right.$ and $\left.\mathrm{C}_{\mathrm{CD}}\right)$, the effective numbers of fibres along MD and CD per $\mathrm{mm}^{2}$ were calculated. The bond points were treated as orthotropic. This approximation helped to simplify the problem by decreasing the number of variables and computation time. Then, the properties of raw materials used for core and sheath of 
the constituent fibres facilitated the calculation of elastic moduli and Poisson's ratio of composite bond points in MD and CD based on the RoM as

$$
\begin{aligned}
& v=v^{\text {core }} V^{\text {core }}+v^{\text {sheath }} V^{\text {sheath }} \\
& E=E^{\text {core }} V^{\text {core }}+E^{\text {sheath }} V^{\text {sheath }}
\end{aligned}
$$

where $v$ and $E$ are the resultant Poisson's ratio and resultant modulus of elasticity; $v^{\text {core,sheath }}$ and $E^{\text {core,sheath }}$ are the Poisson's ratio and elastic moduli and $V^{\text {core,sheath }}$ are volumetric fractions of core and sheath materials, respectively. Along thickness direction (TD), the properties were calculates using Helpin-Tsai equations for composites [21]:

$$
\begin{gathered}
\frac{E_{\mathrm{TD}}}{E_{\text {sheath }}}=\frac{1+2 \eta V_{\text {core }}}{1-\eta V_{\text {core }}}, \\
\eta=\frac{\left(E_{\text {core }} / E_{\text {sheath }}\right)-1}{\left(E_{\text {core }} / E_{\text {sheath }}\right)+2},
\end{gathered}
$$

Where $E_{\text {core }}$ and $E_{\text {sheath }}$ are the elastic moduli of core and sheath materials, respectively. $E_{\mathrm{TD}}$ is the modulus of elasticity in transverse direction and $V_{\text {core }}$ is the volumetric fraction of fibres in composite bond points. The in-plane shear modulus of bond points was calculated by using the following Halpin-Tsai equations [21]:

$$
\begin{gathered}
\frac{G_{\mathrm{LT}}}{E_{\text {sheath }}}=\frac{1+\eta V_{\text {core }}}{1-\eta V_{\text {core }}}, \\
\eta=\frac{\left(G_{\text {core }} / G_{\text {sheath }}\right)-1}{\left(G_{\text {core }} / G_{\text {sheath }}\right)+1},
\end{gathered}
$$

where $G_{\mathrm{LT}}$ is the shear modulus of bond points, $G_{\text {core }}$ and $G_{\text {sheath }}$ are the shear moduli of core and sheath materials, respectively. The orthotropic elastic mechanical properties of the bond points analysed using the input parameters mentioned in Table 2, are given in Table 3. 
Table 3. Computed mechanical properties of bond points

\begin{tabular}{lccc}
\hline Direction & $\begin{array}{c}\text { Young's modulus } \\
\text { (MPa) }\end{array}$ & $\begin{array}{c}\text { Poisson's } \\
\text { ratio }\end{array}$ & $\begin{array}{c}\text { Shear modulus } \\
\text { (MPa) }\end{array}$ \\
\hline Machine Direction & $E_{\mathrm{MD}}=604.9$ & $v_{\mathrm{MD}, \mathrm{CD}}=0.42$ & $G_{\mathrm{MD}, \mathrm{CD}}=369.4$ \\
& $E_{\mathrm{CD}}=325.7$ & $v_{\mathrm{CD}, \mathrm{TD}}=0.42$ & $G_{\mathrm{CD}, \mathrm{TD}}=198.9$ \\
Cross Direction & & & \\
& & & \\
Thickness direction & $E_{\mathrm{TD}}=892.8$ & $v_{\mathrm{TD}, \mathrm{MD}}=0.42$ & $G_{\mathrm{TD}, \mathrm{MD}}=545.3$ \\
\hline
\end{tabular}

In order to model the anisotropic mechanical behaviour of nonwoven in plasticity, the required flow curves of the composite bond points were obtained by normalizing the flow curves of the constituent bicomponent fibres of fabric using the orthotropic ratios obtained from ODF as normalization factor. According to the normalization, the flow curve $\left(\sigma_{\mathrm{i}}\right)$ for MD and CD is calculated as follows:

$$
\sigma_{\mathrm{i}}=\sigma_{\mathrm{f}}\left(\frac{C_{\mathrm{i}} n A_{\mathrm{f}}}{t}\right),
$$

where $\sigma_{\mathrm{f}}$ and $A_{\mathrm{f}}$ are the flow curve and cross-sectional area of the fabric; $n$ is the number of the fibres per $1 \mathrm{~mm}^{2}$ fabric area; $C_{\mathrm{i}}$ is the orthotropic ratio related to the principle direction of the flow curve denoted by $i$, and $t$ is the thickness of the region. Finally, the flow curve of bond points in TD is assumed proportional to that in MD according to

$$
\sigma_{\mathrm{TD}}=\sigma_{\mathrm{MD}}\left(\frac{E_{\mathrm{TD}}}{E_{\mathrm{MD}}}\right),
$$


Where $\sigma_{\mathrm{TD}}$ and $\sigma_{\mathrm{MD}}$ are the flow curves, $E_{\mathrm{TD}}$ and $E_{\mathrm{MD}}$ are the elastic moduli of bond points in TD and $\mathrm{MD}$, respectively. $E_{\mathrm{TD}} / E_{\mathrm{MD}}$ represents the ratio of tensile performance in TD to that in MD. Flow curves of bond points assessed with the developed algorithm are given in Fig. 7. The deformation behaviour of the thermally bonded nonwoven fabric in this study is mainly governed by plasticity; therefore, the curves obtained by the algorithm are in plastic strain range. The properties of the composite bond points would be effected by the core/sheath material and manufacturing parameters. The developed program being fully parametric accounts for this.

The flow chart of the process of obtaining inputs for the FE model is shown in Fig. 8. The development of the discrete phase FE model is given in the next section.

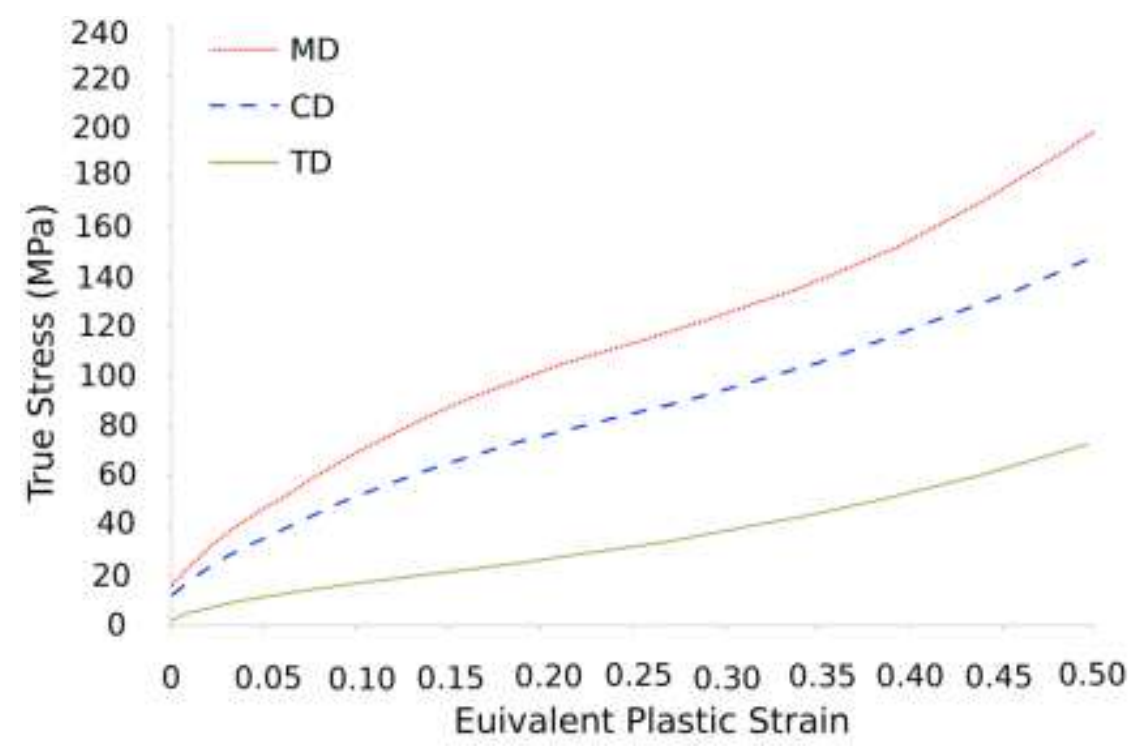

Fig. 7. Computed flow curves of bond points for MD, CD and TD 


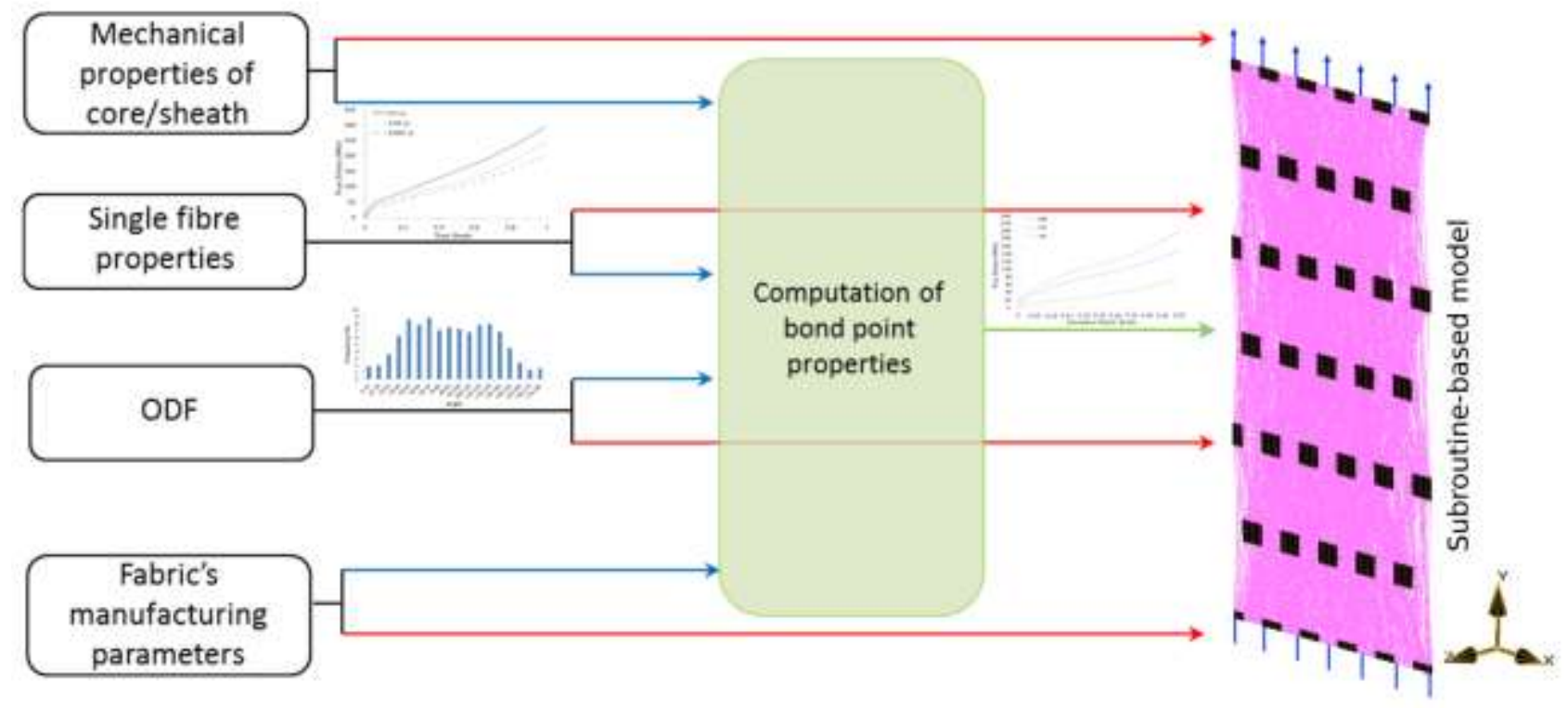

Fig. 8. Flow chart of computing properties of composite bond point

\section{Finite-element Model}

\subsection{Discrete phase model}

A discrete phase finite-element model of thermally bonded nonwoven was developed, which takes into account experimentally observed material's microstructure. The FE model developed in this study comprised two distinct regions - bond points and fibour matrix. Modelling process was started by generating the composite bond points according to their shape and pattern. These bond points form the continuous domain of the model. Then fibres were introduced directly between those bond points according to their orientation distribution function. The approach of direct modelling of fibres in the FE model naturally introduced voids and gaps into it, reflecting the real structure of textile composite fabric. Due to these voids and gaps, the fibrous matrix between bond points forms the discontinuous domain of the model. The number of fibres modelled between the bond points were equal to that in the fabric's coupon with dimensions equal to those of the FE model. To calculate the number of fibres to be modelled, first the total length of fibres in the fabric sample with dimensions equal to those of the FE model was calculated with the following relation:

$$
L_{\text {Fibre_total }}=\frac{m_{\text {Fibre }}}{\rho A} \text {, }
$$


where $\rho$ is the fibre material density, $A$ is the fibre's cross-sectional area, $m_{\text {Fibre }}$ is the mass of fibres within the modelled part of fabric. $m_{\text {Fibre }}$ was calculated by multiplying the fabric density (20 g/m $\mathrm{m}^{2}$ in this study) with the surface area of the modelled fabric sample. Then, the number of fibres to be modelled was calculated by the following relation:

$$
N_{\text {Fibre }}=\frac{L_{\text {Fibre_total }}}{L_{\text {Single_fibre }}},
$$

where $N_{\text {Fibre }}$ is the number of fibres to be modelled and $L_{\text {single_fibre }}$ is the length of a staple fibre. The details of calculation of the number of fibres are given in $[15,22]$. The process of generation of the model with bond points connected by the fibres between them according to their orientation distribution was performed with a subroutine written in Patran Command Language (PCL). This subroutine based on the parametric modelling technique makes it easy to reformulate the model to incorporate the variation in size, shape or pattern of bond points or orientation distribution of fibres. The steps involved in the subroutine for the modelling process are shown in Fig. 9.

The finite-element model was implemented in this study using MSC. Marc software. Bond points were modelled with shell elements (element type 139 in MSC. Marc ${ }^{\circledR}, 2010$ ) with thickness identical to those in the fabric. On the other hand, fibres were modelled as truss elements (element type 9 in MSC. Marc $\left.{ }^{\circledR}, 2010\right)$. The appropriate material properties of fibres and bond point were assigned to the corresponding elements. These material properties were validated before modelling the deformation behaviour if nonwovens, detail given elsewhere [16-17]. Due to anisotropic nature of the fabric caused by the preferential orientation distribution of fibres, two models - along MD and $\mathrm{CD}$ - were prepared; the length of the modelled specimens was $15 \mathrm{~mm}$, their width was $10 \mathrm{~mm}$. The geometry and pattern of the bond points and fibrous matrix connecting them in the model were similar to those of the actual fabric as shown in Fig. 10.

Boundary conditions appropriate for uni-axial tensile testing were applied in the model (Fig. 11). In order to compare and validate the numerical results with experimental data, the boundary conditions 
implemented in the FE model were identical to those used in the tensile tests. The transverse contraction of the fabric was constrained at both ends $-\mathrm{A}-\mathrm{b}$ and $\mathrm{X}-\mathrm{Y}$ in Fig. 11 and $12-$ to simulate the grip effects. Nodes on A-B side were fixed whereas a constant engineering strain rate of 1.0 1/s was applied to the nodes on X-Y side (Fig. 11 and 12).

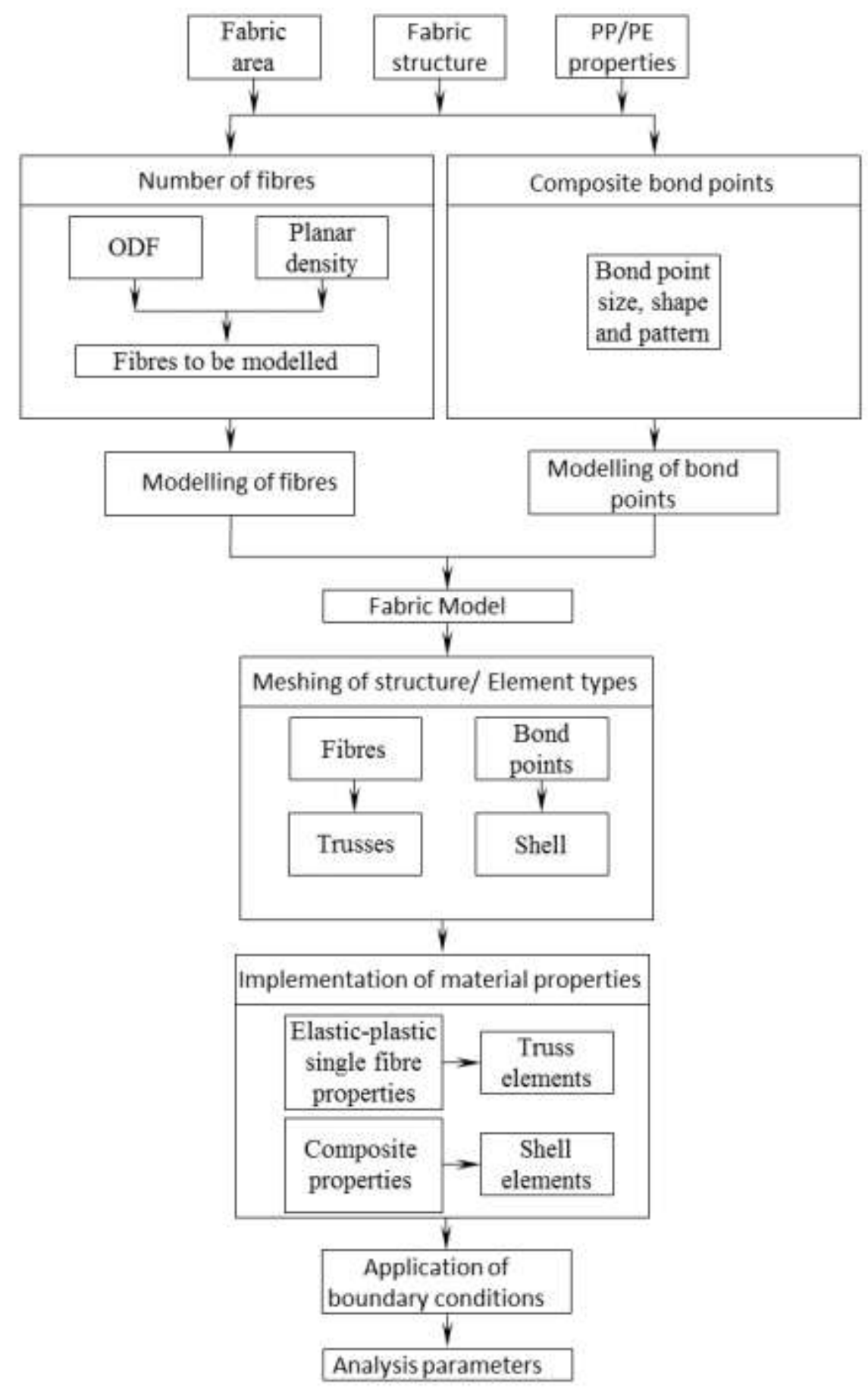

Fig. 9. Flow chart showing steps of subroutine 


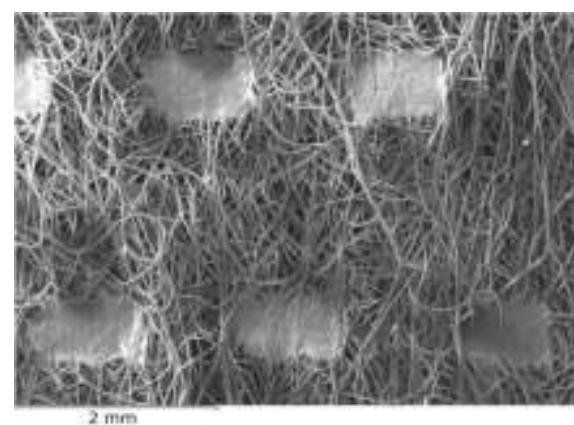

(a)

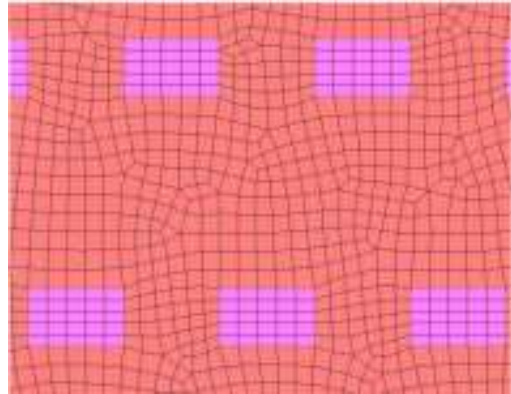

(b)

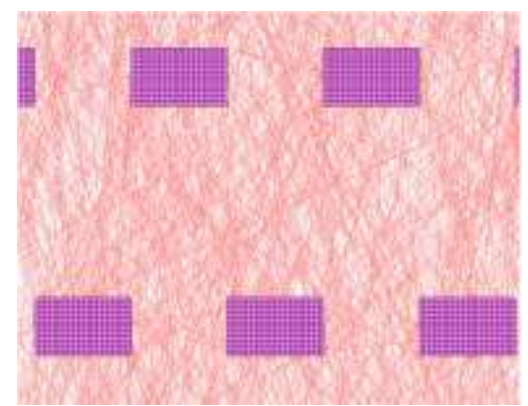

(c)

Fig. 10. (a) SEM image of bicomponent nonwoven; (b) corresponding continuous phase model; (c) discrete phase model

\subsection{Continuous phase model}

In order to better understand the capabilities of the novel discrete phase model, its results were compared with those obtained with a continuous phase FE model developed in [20]. The model is briefly described here. In this model, the entire speicmen (including bond points and fibrous matrix) of the studied nonwoven is meshed with four-node thin shell elements. Appropriate thickness of bond points and fibrous matrix, obtained from CT scan images of the fabric, was assigned to the corresponding elements in the model in a way that it replicates the real fabric structure as shown in Fig. 10b. Orthotropic mechanical properties obtained with a specially developed algorithm, as mentioned in Section 4.1, defined the material behaviour of shell elements used to model bond points. The randomness of fibres orientation between bond points is considered by this algorithm while computing the material properties. The properties of fibrous matrix region were obtained by using the same RoM as in the bond points. However, the behaviour of matrix region in thickness direction was computed by considering a very small nonzero thickness. The flow curves for this region were obtained by normalizing the flow curve of single fibres using the orthotropic ratios obtained from the ODF as normalization factor. The complete details of computation of properties for both regions — bond points and fibrous matrix — are given in [20].

To allow comparability of results of this model with that of discrete phase model and tensile test data, the dimensions and boundary conditions for both models were identical (Fig. 11 and 12). 


\section{Results, validation of model and discussion}

The developed models were used to simulate uni-axial tensile tests in both MD and CD and the obtained results were compared with the experimental data for validation. Figure 13 shows the force-displacement curves calculated with both the models for stretching along MD and CD. The results obtained with the continuous phase model were in good agreement with the experimental data in terms of the force-displacement curve. The slight variation in the models' prediction and experimental data can be attributed to some secondary factors not accounted in the model. The major of these parameters were crimp in fibres and variation in diameter along the length of fibres.

In the simulation strategy based on modelling the fabric as continuum, explicit introduction of fibres was not possible. Thus, the model was unable to predict distribution of the stresses and strains in fibres, which is very important from the industrial point of view to produce more robust nonwovens. Besides, the random pattern of network's deformation resulting in misalignment of bond points, as shown in Fig. 4, could not be reproduced, leading to inaccurate prediction of stresses in composite bond points. Instead, as expected, a specific pattern of bond points arrangement was observed during fabric's deformation, which was symmetric both with respect to the longitudinal axis and the line of maximum transverse contraction of the fabric shown by a yellow line in Fig. 12a. Also, the pattern of stress distribution in bond points demonstrated the same symmetry as shown in Fig. 12b.

The FE model developed employing the discrete phase simulation strategy predicted the mechanical performance of the fabric very well, which is obvious from Fig. 13. The simulation results obtained with discrete phase models are less stiffer than those with continuous phase models and, hence, provide a better agreement with experimental data for both directions. This is explained by a better reproduction of the underpinning mechanism of the initial stages of deformation: rotation of fibres towards the direction of stretching. Apparently, this mechanism cannot be incorporated directly in the continuous phase models. Still the models predicted slightly stiffer 
initial behaviour than the one observed in the experimental results due to secondary factors discussed above.

The shapes of the both types of deformed models were in good agreement with experimental observations. The transverse contraction of the fabric from the grips at its each end increased gradually, and the maximum contraction was occurred at the specimen's centre. However, the reorientation of fibres along the loading direction during fabric's deformation, similar to the observed in experiments, was obtained only by discrete phase model as shown in Fig. 14. This reorientation of fibres connecting the bond points of the fabric resulted in random displacements of bond points as shown in Fig. 11. Once the fibres were aligned along the loading direction, they underwent stretching resulting in stresses in bond points connected to them. Continuous phase model showed almost similar level of transverse contraction in both MD and CD as shown in Fig. $14 \mathrm{a}$ and $14 \mathrm{~b}$ which is not in good agreement with experimental observations. However, discrete phase model showed more realistic transverse contraction. Nevertheless, transverse contraction observed in discrete phase model started to deviate from experimental results as shown in Fig. 14b. The potential reason for this deviation is that some of the fibres were along MD which braces the nonwoven structure against transverse contraction. Due to the stiffer structure of the composite bond points, stresses were more concentrated in these regions. Each bond point had its own pattern of stress distribution depending upon the initial orientation of fibres connected to it and the position of bond point during deformation of the fabric. In general, strong stress concentration at bond points' edges perpendicular to the loading axes was observed.

The variation in stresses in composite bond points according to their position and orientation of fibres connected to them was studied for the first time using this simulation strategy. Although, failure of bond points during in-plane fabric's deformation is not very frequent, however, it is crucial for the load-bearing capacity of nonwovens. Since several fibres are connected to each bond point. Thus, it is important to understand the stresses and strains in bond points. 
Additionally, the discrete phase model can be used to predict the distribution of stresses in fibres of the fabric. Such a distribution at 50\% extension in MD and CD is given in Fig. 15. The stresses in majority of fibres for $\mathrm{CD}$ are very less (ranging from 10MPa to $50 \mathrm{MPa}$ ) as compared to those for MD (ranging from $110 \mathrm{MPa}$ to $170 \mathrm{MPa}$ ). The reason for this phenomenon is the fabric's microstructure. Since fibres were preferentially aligned along MD, large reorientation of fibres took place, as a result of fabric's extension, before participation in load bearing for CD than MD. Thus, higher stresses in fibres were observed for MD than $\mathrm{CD}$ at any level of fabric's extension. The strength, reliability and durability of nonwoven structure can be improved by modifying the ODF in a way to obtain more uniform stress distribution. As discrete phase models provide information on fibres participating in load bearing at any level of fabric's extension with distribution of stresses in them, which is very important to produce nonwovens robust enough to withstand mechanical loading during their working life, this modelling strategy can be used to optimise the ODF of the fabric for its better strength and robustness. Moreover, it can provide insight of structure-properties relationship of nonwoven fabric. This information cannot be obtained from a continuous model or form the experiments. The discrete phase model can also be extended to predict the behaviour of multilayer textile composites having bi-component nonwoven as reinforcement, which will be our future work. 


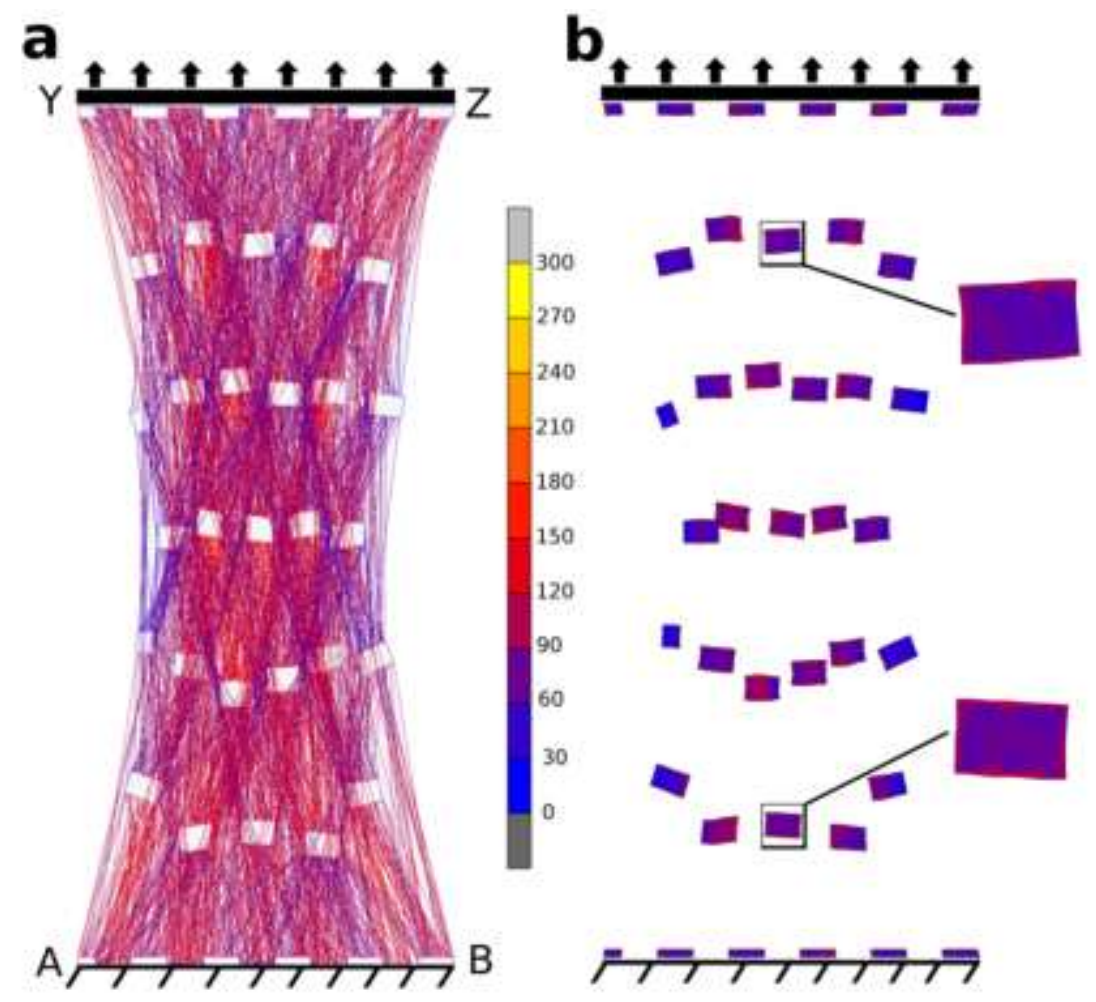

Fig. 11. FE results for discrete phase model at $50 \%$ extension showing equivalent von Mises stresses in (MPa) in MD: (a) regions in deformed model and (b) position of composite bond points

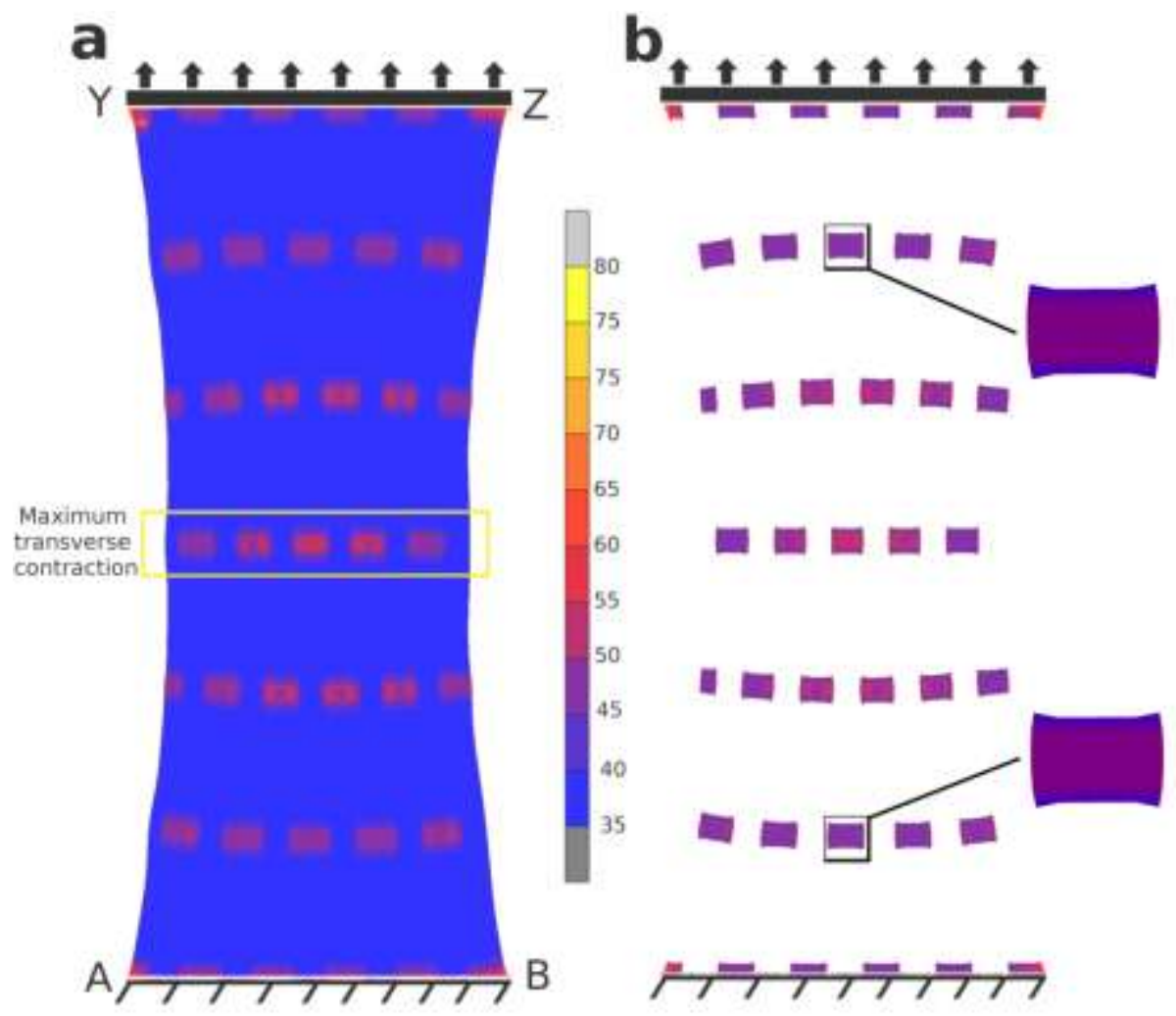

Fig. 12. FE results for continuous model at $50 \%$ extension showing equivalent von Mises stresses in (MPa) in MD: (a) regions in deformed model and (b) position of composite bond points 


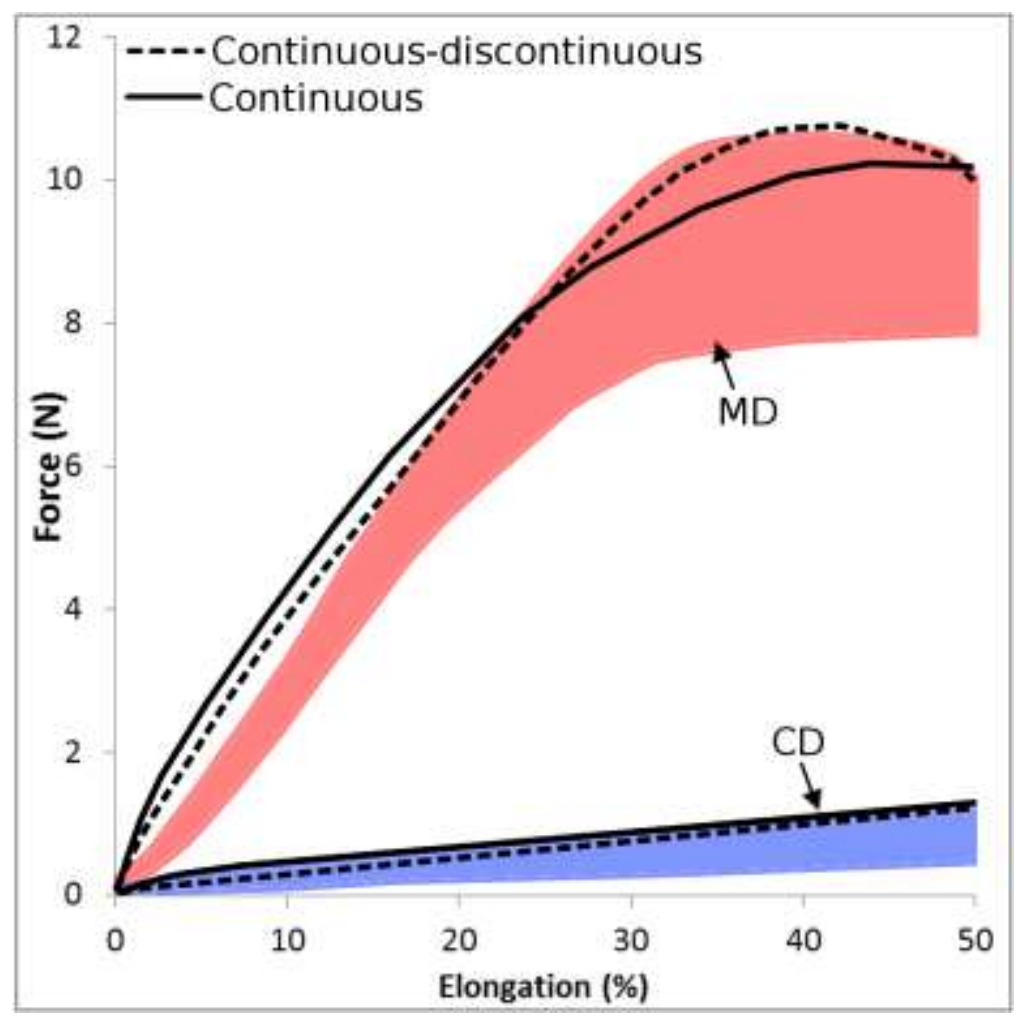

Fig. 13. Force-extension curves from tensile tests and FE simulations in $\mathrm{MD}$ and $\mathrm{CD}$ for PP/PE $35 \mathrm{~g} / \mathrm{m}^{2}$ thermally bonded bi-component fibre nonwoven fabrics (Dimension of specimens: $15 \mathrm{~mm} \times 10 \mathrm{~mm}$ and shaded area represents scatter in experimental results)

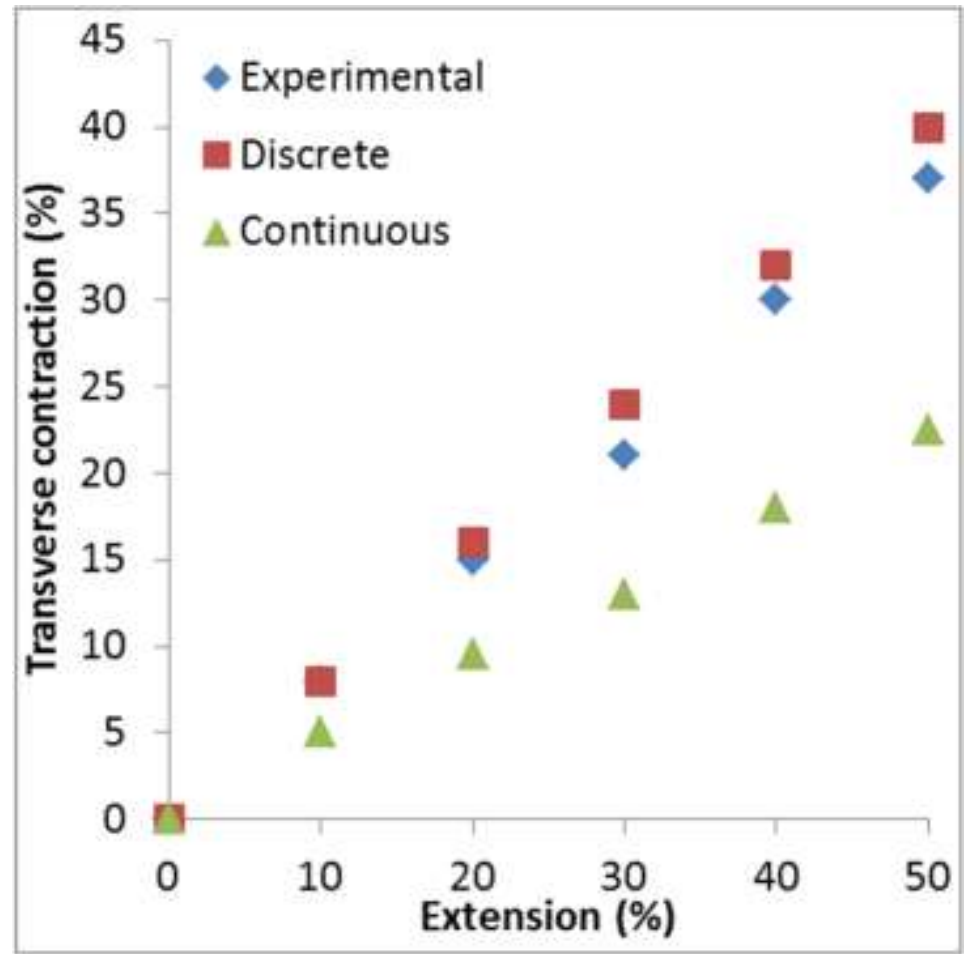

(a) 


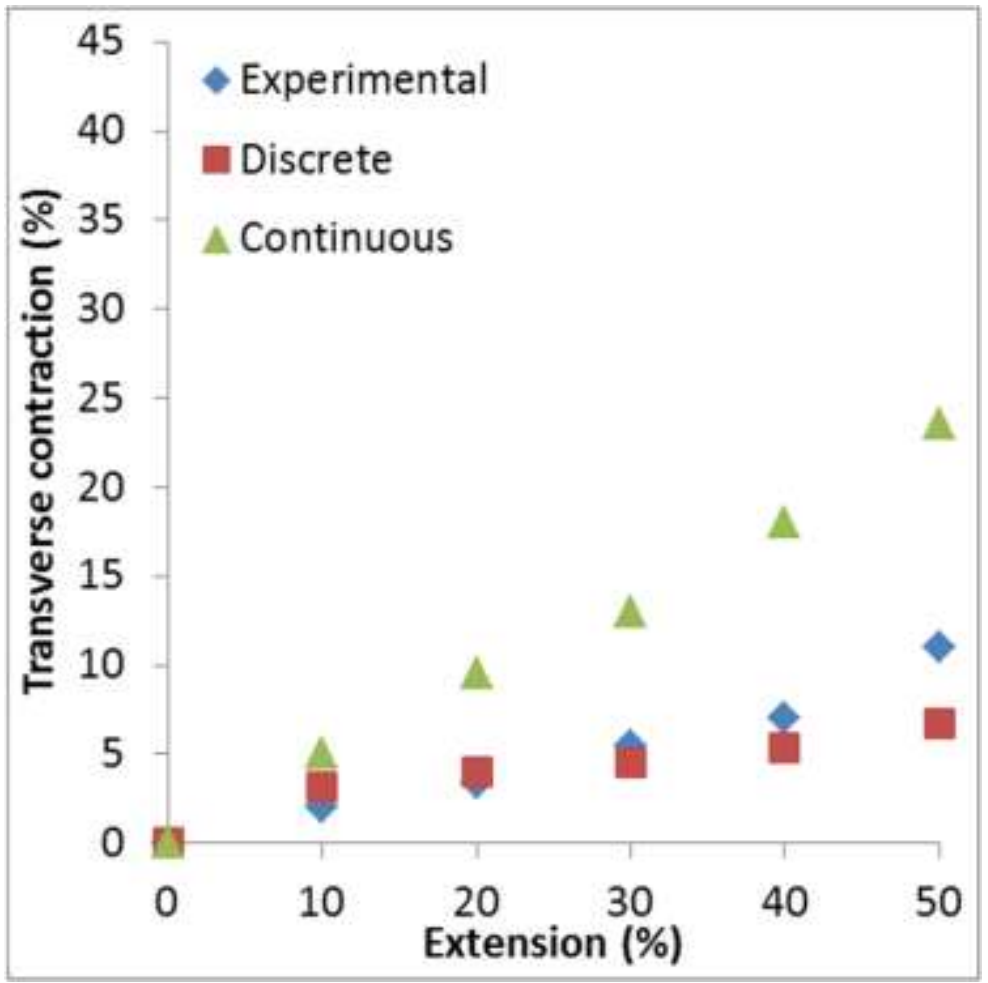

(b)

Fig. 14. Maximum transverse contraction at different fabric's extension in MD (a) and CD (b)

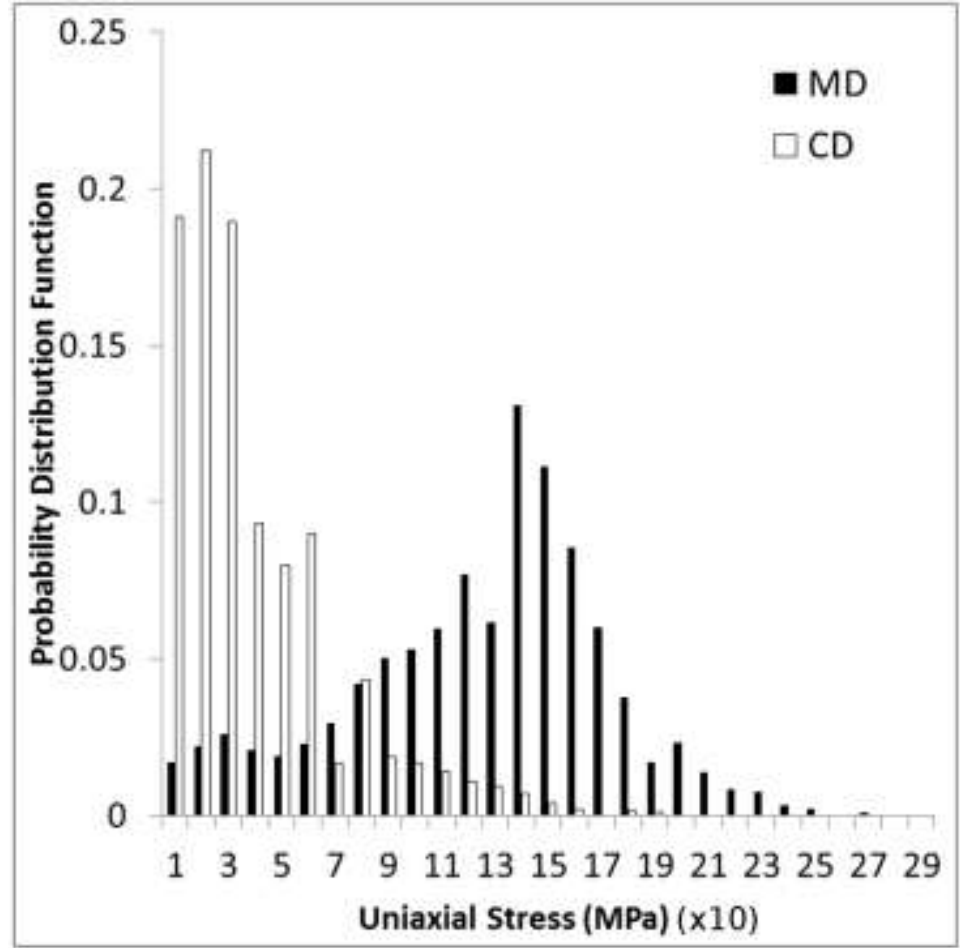

Fig. 15. Distribution of uniaxial stress in fibres in nonwoven structure at $50 \%$ extension 


\section{Conclusions}

A new finite-element simulation strategy is suggested together with a micromechanical computational model developed to represent the anisotropic nonlinear mechanical behaviour of bicomponent fibre nonwoven fabrics. Their composite bond points and fibres connecting these points are treated as two distinct domains with different mechanical properties.

Due to specific microstructure consisting of composites areas connected by randomly oriented fibres, direct introduction of fibres into the model of fibrous network was adopted in this study. This greatly facilitated observations of the stress distribution in each composite area of the fabric (bond point). It was found that each composite bond point had its unique pattern of stress distribution, at all levels of fabric's extension, depending upon its position globally in the structure and orientation of fibres connected to it. The performed uni-axial tensile tests were simulated and their results were compared with those obtained with a previous model based on continuum modelling strategy and the experimental data, demonstrating that the new approach is capable to describe the mechanical behaviour of the nonwoven fabric more precisely. It can also provide the quantitative information about distribution of stresses in fibres of the fabric which could never be obtained from continuous models or from the experiments. As this information is very useful to produce more robust nonwoven structures, this modelling strategy can be used by the industry to produce nonwovens aiming for better strength and reliability. Besides, the successful implementation of simulations of bond points as composites demonstrated that the model can be used to predict the behaviour of composites produced by using the bi-component nonwoven fabric as precursors as mentioned in [7]. Finally, the model can be implemented in any commercial FE software capable to deal with orthotropic material properties.

\section{Acknowledgement}

The authors gratefully acknowledge the support of Nonwovens Cooperative Research Center, North Carolina State University, USA. 


\section{References}

[1] Peng X, Guo Z, Du T, Yu W-R. A simple anisotropic hyperelastic constitutive model for textile fabrics with application to forming simulation. Composites: Part B 2013; 52(275-281).

[2] Russell S. Handbook of Nonwovens. Woodhead Publishing; 2007.

[3] Bhattacharyya D. Composite sheet forming. Elsevier Science; 1997.

[4] Spencer AJM. Deformation of fibre-reinforced materials. Oxford: Clarendon Press; 1972.

[5] Cao J, Akkerman R, Boisse P, Chen J, et al. Characterization of mechanical behavior of woven fabrics: experimental methods and benchmark results. Composites Part A 2008; 39:1037-53.

[6] Albrecht W, Fuchs H, Kittelmann W. Nonwoven fabrics: raw materials, manufacture, applications, characteristics, testing processes. Wiley-VCH Weinheim; 2003.

[7] Desdemir M, Maze B. Formation of novel thermoplastic composites using bicomponent nonwovens as a precursor. J Mater Sci 2011; 46(3269-3281).

[8] Demirci E, Acar M, Pourdeyhimi B, Silberschmidt V V. Finite element modelling of thermally bonded bicomponent fibre nonwovens: Tensile behaviour. Compt Mater Sci 2011; 50:12861291).

[9] Demirci E, Farukh F, Acar M, Pourdeyhimi B, Silberschmidt V V. Numerical analysis of dynamic out-of-plane loading of nonwovens. J Phys: Conf Series 2013; 451(012021).

[10] Sirtautas J. Pickett A K. Lepicier P. A mesoscopic model for coupled drape-infusion simulation of biaxial Non-Crimp Fabric. Composites: Part B 2013; 47:48-57.

[11] Mueller D H. Kochmann M. Numerical Modeling of Thermobonded Nonwovens. Int Nonwovens J 2004; 3:56-62.

[12] Limem S, Warner S B. Adhesive Point-Bonded Spunbond Fabrics. Text Res J 2005; 75:63-72.

[13] Sabuncuoglu B, Acar M, Silberschmidt V V. A parametric finite element analysis method for lowdensity thermally bonded nonwovens. Compt Mater Sci 2012; 52:164-170.

[14] Hou X, Acar M, Silberschmidt V V. Finite element simulation of low-density thermally bonded nonwoven materials: Effects of orientation distribution function and arrangement of bond points. Compt Mater Sci 2011; 50:1292-1298.

[15] Farukh F, Demirci E, Sabuncuoglu B, Acar M, Pourdeyhimi B, Silberschmidt V V. Numerical modelling of damage initiation in low-density thermally bonded nonwovens. Compt Mater Sci 2012; 64:112-115.

[16] Farukh F, Demirci E, Acar M, Pourdeyhimi B, Silberschmidt V V. Meso-scale deformation and damage in thermally bonded nonwovens. J Mat Sci 2013; 48:2334-2345.

[17] Farukh F, Demirci E, Sabuncuoglu B, Acar M, Pourdeyhimi B, Silberschmidt V V. Characterisation and numerical modelling of complex deformation behaviour in thermally bonded nonwovens. Compt Mater Sci 2013; 71:165-171. 
[18] Jinlian H. Fabric Testing. Woodhead Publishing Limited; 2008.

[19] Farukh F, Demirci E, Sabuncuoglu B, Acar M, Pourdeyhimi B, Silberschmidt V V. Numerical analysis of progressive damage in nonwoven fibrous networks under tension. Int J Solids Struct. 2014; 51:1670-1685.

[20] Demirci E, Acar M, Pourdeyhimi B, Silberschmidt V V. Computation of mechanical anisotropy in thermally bonded bicomponent fibre nonwovens. Compt Mater Sci. 2012; 50:157163.

[21] Chou T.W. Microstructural Design of Fiber Composites, Cambridge University Press, Cambridge, $\mathrm{UK} ; 1992$.

[22] Farukh F, Demirci E, Acar M, Pourdeyhimi B, Silberschmidt V V. Mechanical behaviour of nonwovens: Analysis of effect of manufacturing parameters with parametric computational model. Compt Mater Sci. 2014; In press.

[23] MSC. Marc. Volume A: Theory and User Information. 2013. 